1 Universidade Estadual Paulista Júlio de Mesquita Filho (Unesp) - Botucatu (SP), Brasil.

ju.acebal@gmail.com

2 Universidade Federal de São Paulo (Unifesp) - São Paulo (SP), Brasil.

\section{O habitar na reabilitação psicossocial: análise entre dois Serviços Residenciais Terapêuticos}

\author{
Living in psychosocial rehabilitation: analysis between Two \\ Residential Therapeutic Services
}

Júlia Souza Acebal', Guilherme Correa Barbosa', Thiago da Silva Domingos², Silvia Cristina Mangini Bocchi', Aline Tieme Uemura Paiva'

DOI: $10.1590 / 0103-1104202012713$

RESUMO Buscou-se analisar a interlocução das condições e das necessidades de moradia dos usuários em sofrimento psíquico grave dos Serviços Residenciais Terapêuticos em relação à inserção social e à autonomia. Pesquisa transversal realizada com 74 moradores dos Serviços Residenciais Terapêuticos de um município do interior do estado de São Paulo. Foi aplicado Instrumento para Estudos das Condições de Moradias de Portadores de Transtorno Mental Grave. O tratamento dos dados ocorreu por meio do programa Statistical Package Social Sciences, utilizando medidas descritivas e análise estatística com o teste qui-quadrado, considerando Intervalo de Confiança de 95\% e p-valor<0,05. Entre os participantes, predominaram: sexo feminino, cor branca, não alfabetizado, sem parceiros e sem vínculo empregatício, morando mais que dois anos no serviço residencial e, em média, há 38 anos em tratamento psiquiátrico. Identificaram-se diferenças entre os serviços residenciais em relação à inserção social e à autonomia dos usuários e se discute a influência da localização geográfica desses serviços. A centralidade das residências terapêuticas à rede de saúde implica a promoção de inserção, autonomia e satisfação dos usuários em sofrimento psíquico grave. Assim, a fragilidade do habitar compromete a efetivação da reabilitação psicossocial compreendida no tripé: trabalho, rede social e moradia.

PALAVRAS-CHAVE Desinstitucionalização. Saúde mental. Serviços de saúde mental. Pesquisa sobre serviços de saúde. Habitação.

ABSTRACT The objective of this study was to analyze the relation of housing conditions and needs of users with severe mental disorders at therapeutic residential services regarding social inclusion and autonomy. A cross-sectional study was conducted with 74 residents of residential therapeutic services in a city in the countryside of São Paulo. The Instrument for the Study of the Housing Conditions of People with Severe Mental Disorder was applied. Data were processed using the Statistical Package Social Sciences program through descriptive measures and statistical analysis with the chi-square test. A Confidence Interval of $95 \%$ and $p$-value $<0.05$ were considered. There was a predominance of the variables female gender, white, illiterate, without partners and without employment relationship, with users who had been residing for more than two years in the residential service and who were, on average, 38 years under psychiatric treatment. Differences between residential services in relation to social inclusion and autonomy of users were identified and the influence of the geographic location of these services is discussed. The centrality of therapeutic residences within the health network implies the promotion of insertion, autonomy, and satisfaction of residents. Thus, the fragility of housing compromises the effectiveness of psychosocial rehabilitation comprised in the triad work, social network and housing.

KEYWORDS Deinstitutionalization. Health mental. Mental health services. Health services research. Housing. 


\section{Introdução}

A Reforma Psiquiátrica brasileira tem provocado uma transformação no modelo de cuidado em saúde mental. As repercussões desse processo são inúmeras e se distribuem em um continuum cuja cadência é característica de movimentos sócio-históricos e culturais: entre avanços e retrocessos ${ }^{1}$. A abordagem proposta nesta pesquisa se refere aos Serviços Residenciais Terapêuticos (SRT) que constituem dispositivos de cuidado inseridos como equipamento da Rede de Atenção Psicossocial (Raps) e que representam uma implicação central no processo de reabilitação psicossocial de pessoas com transtornos mentais graves e persistentes ${ }^{2}$.

Desse modo, os SRT foram oficializados no Sistema Único de Saúde (SUS) por meio da Portaria $n^{\circ} 106$, de 2000 , publicada pelo Ministério da Saúde. Nesse documento, propõe-se que os SRT sejam casas inseridas, preferencialmente, no espaço urbano para receber até oito egressos de internações prolongadas e oriundos de instituições manicomiais, contando com suporte clínico e psicossocial ${ }^{3}$. Posteriormente, ocorreu a instituição do auxílio-reabilitação psicossocial com o Programa De Volta pra Casa (PVC) sob a Lei $\mathrm{n}^{\circ} 10.708$, de 2003 , regulamentada pela Portaria ${ }^{\circ} 2.077$, de 2003 , destinada para pacientes moradores de SRT e pessoas acometidas por transtornos mentais graves institucionalizadas por um período mínimo de dois anos ininterruptamente ${ }^{4}$.

Em consideração às necessidades de saúde das pessoas portadoras de transtornos mentais graves e persistentes no contexto da desinstitucionalização, são propostas duas modalidades de SRT. O tipo I é destinado a pessoas com internação de longa permanência sem vínculos familiares e sociais. Deve acolher de quatro a oito moradores, que são vinculados a um serviço/equipe de saúde mental de referência para o suporte técnico-especializado necessário ao serviço residencial. $\mathrm{O}$ acompanhamento dos moradores acontece em consonância com os respectivos projetos terapêuticos singulares, sob a lógica da criação de um espaço para construção de autonomia, levando à retomada da vida cotidiana e reinserção social. Tal suporte focaliza-se no processo de reabilitação psicossocial e inserção dos moradores na rede social existente (trabalho, lazer, educação, entre outras). Pode-se contar com um cuidador de referência, cuja incorporação deve ser avaliada pela equipe técnica de acompanhamento do SRT, vinculada ao equipamento de saúde de referência, e ocorrerá mediante a necessidade de cuidados de cada grupo de moradores, levando-se em consideração o número e o nível de autonomia dos moradores ${ }^{5}$.

O tipo II entre os SRT consiste em uma modalidade destinada àquelas pessoas com maior grau de dependência, necessitando de cuidados intensivos e específicos que demandam ações diretivas com apoio técnico diário e pessoal permanente; entre esses, cuidadores de referência e equipe de enfermagem. Acolhe no mínimo quatro e no máximo dez moradores. O encaminhamento destes deve ser previsto a partir do projeto terapêutico singular, elaborado por ocasião do processo de desospitalização, com foco na reapropriação do espaço residencial como moradia, na construção de habilidades para a vida diária, autocuidado, alimentação, vestuário, higiene, formas de comunicação e aumento das condições para estabelecimento de vínculos afetivos, com inserção desses domiciliados na rede social existente. O ambiente doméstico deve considerar adequações e adaptações no espaço físico que melhor atendam às necessidades dos moradores 5 .

A moradia atua como um condicionante social e para a saúde mental, especificamente, torna-se decisiva para inserção, integração e manutenção do usuário com transtorno mental grave no meio social, conformando, com o trabalho e a rede social, o tripé da reabilitação psicossocial ${ }^{6,7}$.

Nos espaços dos SRT, há a necessidade de o cuidado ser construído em um cotidiano assistido, pois a institucionalização nos serviços 
manicomiais provocou a fragmentação dos valores, da autonomia e dos laços familiares. Ressalta-se a importância de uma nova perspectiva de vida, fundamentada no resgate do protagonismo de um sujeito com sua história vivida no território. Em síntese, a dimensão do assistir está centrada na intermediação entre o cuidado e a autonomia, no sentido de ser capaz de reconhecer a fragilidade do sujeito na sua inserção e no resgate de sua atuação nas ações cotidianas intrínsecas ao morar ${ }^{8}$.

Mesmo diante da irrefutabilidade dos avanços provenientes da implantação dos SRT e de outros serviços guiados pela lógica territorial e comunitária para o processo de reordenação da assistência em saúde mental no Brasil, algumas fragilidades são identificadas no processo de trabalho desses serviços e constituem desafios para o trabalho e o cuidado nos espaços das SRT. Dentre estes, destacam-se: o distanciamento das equipes de referências em saúde mental e cuidado centrado na clínica biomédica, repercutindo na ausência de projetos terapêuticos singulares para os moradores; o escopo muito diversificado de atividades dos cuidadores, privilegiando ações concretas e cotidianas em detrimento de ações que objetivem a reabilitação psicossocial e a aquisição de autonomia; e a prática de vigilância e de controle, dificultando a construção de um espaço para o exercício da privacidade 9 .

Além da problemática explicitada, o município abordado nesta investigação encontra-se em processo de ampliação das unidades que constituem o SRT, bem como possui dois Centros de Atenção Psicossocial (Caps) destinados para demanda de pessoas acometidas por transtornos mentais graves e persistentes cuja procura vem extrapolando a oferta de cuidado possível para os serviços. Vale ressaltar que a gestão desses serviços se dá entre as esferas municipal e estadual, configurando uma organização peculiar deles e conferindo uma particularidade que os diferencia e que se reflete no cotidiano do cuidado.

Pelo exposto, apresenta-se como pergunta de pesquisa: 'De que modo as condições e as necessidades de moradia identificadas pelos usuários em sofrimento psíquico grave dos SRT estão relacionadas com a inserção social e com a autonomia?'. Diante desse questionamento, objetivou-se analisar a interlocução das condições e das necessidades de moradia dos usuários em sofrimento psíquico grave dos SRT em relação à inserção social e à autonomia.

\section{Material e métodos}

Trata-se de uma pesquisa transversal do tipo descritiva e analítica. Sua execução propõe verificar determinada exposição (condições e necessidades ao residir em SRT) em dois grupos de participantes similares e seus respectivos desfechos (inserção social e autonomia) em um único momento ${ }^{10}$.

A coleta de dados foi realizada em um município do interior do estado de São Paulo/ Brasil. No que se refere à Raps, conta com 20 Unidades Básicas de Saúde, uma unidade de Serviço de Atendimento Móvel de Urgência, dois Hospitais Gerais, sendo que um deles possui uma unidade de internação psiquiátrica com seis leitos, e um Serviço Referência em Álcool e Outras Drogas que funciona em caráter de internação. O Centro de Atenção Integral à Saúde oferece uma unidade de internação psiquiátrica com leitos para pessoas com transtornos mentais agudos, um Caps tipo II, um Caps Álcool e Drogas, uma Oficina Terapêutica e um STR. Além disso, a gestão municipal possui também um STR e um Caps tipo I. Como estratégia de reabilitação psicossocial, cita-se a 'Arte Convívio', que é um equipamento de economia solidária que funciona na lógica de cooperativa.

Incluíram-se todos os seis SRT do município e seus respectivos moradores. Sob gestão da esfera estadual, havia três residências terapêuticas, sendo duas do tipo I e uma do tipo II, nas quais residiam 33 moradores; inseridas em um complexo assistencial distante da área urbana, aproximadamente, sete quilômetros do centro comercial. Assim, 
sem nenhum tipo de comércio e serviços que pudesse ser frequentado pelos moradores, situação que levava ao estreitamento do convívio social encerrado aos trabalhadores, aos familiares e aos demais moradores. $\mathrm{O}$ apoio técnico especializado era oferecido pelo Caps II, que integrava o referido complexo assistencial, e por uma Unidade Saúde da Família, administrada pela gestão municipal.

Outras três moradias, classificadas como tipo I, estavam sob responsabilidade da administração municipal e possuíam 41 moradores. Sua inserção geográfica estava distribuída entre bairros da área urbana, possibilitando maior integração social ao cotidiano de pessoas que não possuem transtorno mental grave, próximas do comércio e de locais públicos, permitindo a circulação dos referidos moradores. $\mathrm{O}$ apoio técnico especializado, por sua vez, era oferecido pelo Caps I e pela Unidade Básica de Saúde, ambos administrados pela gestão municipal.

No estudo, são comparados os desfechos autonomia e inserção social em função da localização geográfica das residências terapêuticas, que varia do completo isolamento social, identificadas pela sigla SRT1, à inserção em bairros comuns do município, por sua vez, denominadas pela sigla SRT2.

A amostra foi constituída da totalidade dos 74 usuários atendidos pelos respectivos serviços, que preencheram os seguintes critérios de inclusão: 1) ser munícipe da cidade de estudo; 2) residir em alguma moradia do SRT; e 3) demonstrar interesse em participar da pesquisa. Não foram elencados critérios de exclusão. Quando identificada presença de deficit cognitivo severo do participante que comprometia responder ao instrumento de coleta de dados, foi solicitado auxílio do cuidador do integrante para responder aos instrumentos de coleta de dados. Parte das informações foi acessada por meio dos prontuários dos participantes que foram consultados nos respectivos Caps que os atendiam.

Para coleta de dados, aplicou-se o 'Instrumento para Estudos das Condições de Moradias de Portadores de Transtorno Mental Grave'. Trata-se de um questionário elaborado para a investigação de dois aspectos centrais do conceito de moradia para o sujeito em sofrimento psíquico grave no contexto da desinstitucionalização, que são aspectos da vida material e social. É constituído de 71 itens distribuídos em 6 blocos: 1 ) Identificação; 2) Aspectos do quadro clínico; 3) Socioeconômico; 4) Características do imóvel; 5) Inserção social e Autonomia; e 6) Habitação e Satisfação"1.

Os dados foram analisados por meio do programa Statistical Package for Social Sciences, versão 13.0, envolvendo a estatística descritiva (frequências absolutas e relativas, medidas de tendência) e analítica, com o teste qui-quadrado, considerando intervalo de confiança de $95 \%$ e valor de $\mathrm{p}<0,05$.

O estudo foi aprovado pelo Comitê de Ética em Pesquisa da Faculdade de Medicina de Botucatu, recebendo o parecer favorável em 7 de junho de 2018, sob o no 2.699.279. Foi adotado o Termo de Consentimento Livre e Esclarecido destinado para o responsável legal pelo participante, bem como o Termo de Assentimento Livre e Esclarecido para o participante da pesquisa.

\section{Resultados}

No que se refere às características sociodemográficas, observou-se que a média etária foi de 65,5 anos (34-88 anos). Houve predominância para o sexo feminino, cor branca, sem alfabetização, solteiros, desempregados e com tempo de moradia no SRT maior que dois anos. A tabela 1 detalha as frequências absolutas e relativas das variáveis sociodemográficas. 
Tabela 1. Caracterização do perfil sociodemográfico dos participantes da pesquisa. Botucatu/SP, Brasil, 2019. ( $n=74)$

\begin{tabular}{|c|c|c|}
\hline Variáveis & $\mathbf{n}$ & $\%$ \\
\hline \multicolumn{3}{|l|}{ Sexo } \\
\hline Masculino & 35 & $47,3 \%$ \\
\hline Feminino & 39 & $52,7 \%$ \\
\hline \multicolumn{3}{|l|}{ Cor } \\
\hline Branca & 42 & $56,8 \%$ \\
\hline Preta & 17 & $23,0 \%$ \\
\hline Amarela & 12 & $16,2 \%$ \\
\hline Parda & 3 & $4,1 \%$ \\
\hline \multicolumn{3}{|l|}{ Escolaridade } \\
\hline Alfabetizado & 23 & $31,1 \%$ \\
\hline Não alfabetizado & 51 & $68,9 \%$ \\
\hline \multicolumn{3}{|l|}{ Situação conjugal } \\
\hline Solteiro & 72 & $97,3 \%$ \\
\hline Viúvo & 1 & $1,4 \%$ \\
\hline Divorciado & 1 & $1,4 \%$ \\
\hline \multicolumn{3}{|l|}{ Situação profissional } \\
\hline Desempregado & 68 & $91,9 \%$ \\
\hline Aposentado & 2 & $2,7 \%$ \\
\hline Assalariado sem registro na carteira & 4 & $5,4 \%$ \\
\hline \multicolumn{3}{|l|}{ Tempo que reside no SRT } \\
\hline Maior ou igual a 24 meses & 64 & $86,5 \%$ \\
\hline Entre 12 e 24 meses & 8 & $10,8 \%$ \\
\hline Menor que 6 meses & 2 & $2,7 \%$ \\
\hline
\end{tabular}

Fonte: Elaboração própria.

Quanto aos aspectos financeiros, identificou-se que $81,1 \%(n=60)$ recebiam algum tipo de benefício do governo, sendo eles o Benefício de Prestação Continuada (BPC) e o PVC. Verificou-se que a totalidade dos participantes tinha uma parcela do valor recebido destinada para manter a moradia e seus gastos domiciliares, o acesso ao restante do valor era intermediado e controlado, dificultando ao usuário ter autonomia para administrar o seu recurso financeiro. A respeito da quantidade de moradores por casa, observou-se uma média de 7 usuários, variando de 1 a 10, gerando para o grupo uma renda média de $\mathrm{R} \$ 713,90$ ( $\mathrm{R} \$$ $240,80$ a $R \$ 1.020,80)$. Por fim, uma parcela expressiva, 67,6\% ( $\mathrm{n}=50)$, afirmou receber doações ou ajuda de terceiros.

Para descrever o perfil clínico, utilizou-se o diagnóstico médico baseado na Classificação Estatística Internacional de Doenças e Problemas Relacionados à Saúde (CID-10). Houve maior frequência para o grupamento F20-F29 - Esquizofrenia, transtornos esquizotípicos e delirantes, registrado para $54,05 \%(\mathrm{n}=40)$ dos participantes. O segundo grupamento mais prevalente correspondeu a F70-F79 - Retardo mental, para 32,43\% ( $\mathrm{n}=24)$ dos participantes. Em seguida, o grupamento F10-F19 - Transtornos devidos ao uso de substância psicoativa, com $4,1 \%$ dos participantes 
$(n=3)$. Com a mesma frequência, o diagnóstico de outros transtornos mentais especificados devido a uma lesão ou disfunção cerebral e a uma doença física (F06.8). Dois participantes $(2,7 \%)$ diagnosticados com transtorno de personalidade histriônica (F60.4). Um participante com transtorno afetivo bipolar (F31.2) e outro com transtorno mental orgânico (F09).

O tempo de tratamento psiquiátrico teve média de 38 anos, variando de 1 a 64 anos - nesse período, os participantes relataram quantidade de internações variando de 1 a 16 vezes, com média em 2 internações. A totalidade referiu ter sido internada em hospital psiquiátrico; 20,3\% ( $n=15)$, em Caps III; e 1,4\% ( $n=1)$, internação em clínica particular. Em contrapartida, $94,6 \%(n=70)$ negaram internação em hospital geral. Não houve ocorrências de internação em comunidade terapêutica. Todos os usuários referiram tratamento com psicofármacos e acompanhamento por profissional ou uma equipe no Caps. Destes, 95,9\% (n=71) relataram a percepção de poder contar com auxílio de um profissional ou equipe do Caps.

A noção da gravidade do problema de saúde mental foi relatada por $86,5 \%$ dos participantes
( $\mathrm{n}=64)$. A maioria dos usuários $(79,7 \%, \mathrm{n}=59)$ afirmou que seu problema de saúde mental influencia muito negativamente nas atividades do cotidiano. Uma parcela menor apontou pouca $(9,5 \%, \mathrm{n}=7)$, muito pouca $(6,8 \%, \mathrm{n}=5)$ ou nenhuma influência (4,1\%, n=3). Entre as atividades mais influenciadas, a relação com familiares foi mais frequentemente mencionada e relatada por $86,5 \%$ $(\mathrm{n}=64)$ dos participantes, seguida da moradia $(75,7 \%, \mathrm{n}=56)$, relacionamento com outras pessoas $(71,6 \%, \mathrm{n}=53)$, lazer $(62,2 \%, \mathrm{n}=46)$, e, por fim, trabalho e estudos $(23 \%, n=17)$. A relação com namorado(a) foi mencionada por uma minoria, $6,8 \%(n=5)$ dos participantes.

A tabela 2 apresenta a comparação entre os participantes dos dois SRT no que se refere às atividades que perceberam ser influenciadas pela gravidade do transtorno mental. Não houve diferença estatística nas variáveis relacionamento familiar $(\mathrm{p}=0,712)$ e conjugal $(p=0,651)$. Observa-se que os participantes do SRT1 perceberam maior influência nos aspectos do lazer, moradia e relacionamentos com outras pessoas, enquanto os participantes do SRT2 apontaram o trabalho e os estudos, diferenças estas com significância estatística.

Tabela 2. Análise estatística das atividades influenciadas pelo transtorno mental grave. Botucatu (SP), Brasil. 2019. ( $n=74)$

\begin{tabular}{|c|c|c|c|c|c|}
\hline \multirow[b]{2}{*}{ Variáveis } & \multicolumn{2}{|c|}{ SRT $1(n=33)$} & \multicolumn{2}{|c|}{ SRT $2(n=41)$} & \multirow[b]{2}{*}{$\mathrm{p}$-valor } \\
\hline & $\mathbf{N}$ & $\%$ & $\mathbf{N}$ & $\%$ & \\
\hline Trabalho & 4 & $12,12 \%$ & 13 & $31,71 \%$ & 0,056 \\
\hline Estudos & 4 & $12,12 \%$ & 13 & $31,71 \%$ & 0,056 \\
\hline Lazer & 29 & $87,88 \%$ & 17 & $41,46 \%$ & $<0,001$ \\
\hline Moradia & 30 & $90,91 \%$ & 26 & $63,41 \%$ & 0,007 \\
\hline Relacionamento com outras pessoas & 31 & $93,94 \%$ & 22 & $53,66 \%$ & $<0,001$ \\
\hline
\end{tabular}

Fonte: Elaboração própria.

Ainda acerca do perfil clínico, identificou-se que $71,6 \%(n=53)$ dos participantes responderam afirmativamente para comorbidade, que foram distribuídas segundo sistemas corporais acometidos, entre esses, os sistemas cardiovascular e endócrino foram mais prevalentes, ao serem identificados, respectivamente, em $43,24 \%(n=32)$ e $36,48 \%$ ( $n=27)$ da população. Os demais sistemas foram: respiratório $(5,40 \%$, $\mathrm{n}=4)$, locomotor $(1,35 \%, \mathrm{n}=1)$, nervoso $(6,77 \%$, $\mathrm{n}=5)$, imunológico $(4,05 \%, \mathrm{n}=3)$ e gastrointestinal $(6,77 \%, n=5)$. 
Sobre a condição legal da moradia, $62,2 \%$ $(n=46)$ referiram ter a casa cedida pelo governo ou pela instituição, enquanto $37,8 \%(n=28)$ possuem a casa alugada, sendo o custo rateado entre os moradores. Quanto à estrutura, 79,8\% $(n=59)$ residiam em casas de quatro ou cinco cômodos. Todas as moradias se encaixaram em uma edificação do tipo isolada, e, em média, cada participante dividia o quarto com um outro usuário; contudo, isso variou entre não dividir ou dividir com outros três.

Com relação aos lugares próximos (conseguir ir andando), todos afirmaram residir perto de algum serviço de saúde (hospital, pronto-socorro, Unidade Básica de Saúde ou Caps). Aproximadamente metade da população $(52,7 \%, n=39)$ referiu estar próxima a algum serviço da educação (creches ou escolas); e serviços de comércio (agência bancária, comércio em geral, padaria, açougue, farmácia, quitanda, mercearia, supermercado e bares) foram mencionados por $55,4 \%(n=41)$. Locais para entretenimento (centro comunitário, clubes e praças) foram citados com menor frequência $(37,8 \%, \mathrm{n}=28)$ entre os participantes, assim como locais para expressão religiosa $(32,4 \%, n=24)$. Todos negaram a proximidade de serviços de segurança (posto policial e delegacia) ou assistência social - Centro de Referência da Assistência Social (Cras).

A tabela 3 apresenta a comparação entre os participantes dos dois SRT no que se refere ao bloco inserção social e autonomia do instrumento de coleta de dados. Em geral, os participantes da SRT2 possuíam maior disponibilidade de/e frequência aos serviços próximos à moradia, tinham maior contato com estranhos e maior independência nas atividades do cotidiano. Em contrapartida, os participantes da SRT1 recebiam mais visitas.

Tabela 3. Análise estatística das variáveis inserção social e autonomia dos participantes segundo SRT. Botucatu (SP), Brasil, 2019. $(n=74)$

\begin{tabular}{|c|c|c|c|c|c|}
\hline \multirow[b]{2}{*}{ Variáveis } & \multicolumn{2}{|c|}{ SRT $1(n=33)$} & \multicolumn{2}{|c|}{ SRT $2(n=41)$} & \multirow[b]{2}{*}{ p-valor } \\
\hline & $\mathbf{N}$ & $\%$ & $\mathbf{N}$ & $\%$ & \\
\hline \multicolumn{6}{|l|}{ Serviços próximos à moradia } \\
\hline Educação & 33 & $100 \%$ & 6 & $14,63 \%$ & $<0,001$ \\
\hline Comércio & 0 & $0 \%$ & 41 & $100 \%$ & $<0,001$ \\
\hline Entretenimento & 0 & $0 \%$ & 28 & $68,29 \%$ & $<0,001$ \\
\hline Religião & 0 & $0 \%$ & 24 & $58,54 \%$ & $<0,001$ \\
\hline \multicolumn{6}{|c|}{ Serviços próximos à moradia que frequenta } \\
\hline Entretenimento & 2 & $6,06 \%$ & 11 & $26,83 \%$ & 0,030 \\
\hline Comércio & 7 & $21,21 \%$ & 24 & $58,54 \%$ & 0,001 \\
\hline \multicolumn{6}{|c|}{ Pessoas com quem conversa fora da moradia } \\
\hline Desconhecidos & 0 & $0 \%$ & 12 & $29,27 \%$ & $<0,001$ \\
\hline \multicolumn{6}{|l|}{ Pessoas que o visitam na moradia } \\
\hline Familiares & 7 & $21,21 \%$ & 2 & $4,88 \%$ & 0,032 \\
\hline Vizinhos & 11 & $33,33 \%$ & 0 & $0,0 \%$ & $<0,001$ \\
\hline Pessoal do abrigo & 8 & $24,24 \%$ & 1 & $2,44 \%$ & 0,009 \\
\hline Ninguém & 16 & $48,48 \%$ & 38 & $92,68 \%$ & $<0,001$ \\
\hline \multicolumn{6}{|c|}{ Atividades que realiza sozinho e sem ajuda } \\
\hline Higiene Pessoal & 16 & $48,48 \%$ & 30 & $73,17 \%$ & 0,029 \\
\hline Ir a locais de interesse na cidade & 0 & $0,0 \%$ & 13 & $31,71 \%$ & $<0,001$ \\
\hline Tomar medicação & 0 & $0,0 \%$ & 8 & $19,51 \%$ & 0,007 \\
\hline
\end{tabular}

Fonte: Elaboração própria. 
Dos serviços que ficam próximos à moradia, não houve diferença estatística entre os SRT analisados para as variáveis serviços de segurança e assistência social. A mesma análise ocorreu sobre os lugares que os participantes frequentaram: todos afirmaram ir a serviços de saúde ( $\mathrm{p}=1,000)$; nenhum dos participantes de ambos os SRT frequentou serviços de segurança, da educação ou locais para expressão religiosa ( $\mathrm{p}=1,000)$; dois de cada SRT frequentaram o Cras $(\mathrm{p}=1,000)$.

A despeito do local de moradia, os participantes conversam com vizinhos, com outros usuários ou profissionais do Caps ou do SRT, com colegas de trabalho ou comerciantes. Não houve ocorrência para pessoas do meio religioso ou namorados(as). Para ambas as situações, não houve diferença estatística com valores de $\mathrm{p}>0,05$.

Também não houve diferença estatística para a pergunta 'quando você tem um problema e precisa de ajuda, quem você procura?': familiares $(\mathrm{p}=0,195)$; usuários de outros serviços de saúde ( $\mathrm{p}=1,000)$; pessoal da igreja $(\mathrm{p}=1,000)$; pessoal da prefeitura $(\mathrm{p}=1,000)$; usuários do Caps $(\mathrm{p}=1,000)$; vizinhos $(\mathrm{p}=0,084)$; pessoal do abrigo $(\mathrm{p}=0,499)$; colegas de trabalho ( $\mathrm{p}=1,000)$; ninguém $(\mathrm{p}=0,249)$.

Não houve relatos por parte dos participantes de receberem visitas de usuários de outros serviços de saúde, pessoal da igreja, pessoal da prefeitura, usuários do Caps ou colegas de trabalho. Todos referiram receber visitas de profissionais de saúde de algum serviço da atenção básica ou psicossocial estratégica. Para ambos os casos, não houve diferenças estatísticas ( $p>0,05)$.

No que diz respeito às atividades que o participante conseguia fazer sozinho, $4,88 \%(n=2)$ referiram conseguir ir ao banco para receber seu dinheiro, e 9,76\% $(n=4)$, realizar compras e pagamentos, todos moradores da SRT2 ( $n=41)$ - não havendo diferença estatística entre os SRT, com $\mathrm{p}$-valores, respectivamente, $\mathrm{p}=0,124$ e $\mathrm{p}=0,449$.

Por fim, verificou-se o grau de satisfação dos participantes em relação aos SRT em que residiam. Aponta-se uma maior satisfação entre os participantes do SRT2, uma vez que o escore geral foi de $33(\mathrm{DP} \pm 31-43)$ para os participantes do SRT1 e 36 (DP $\pm 31-51)$ para aqueles do SRT2. A análise dessa variável apontou diferença estatística com valor de $\mathrm{p}=0,039$. O gráfico 1 detalha cada um dos fatores analisados.

Gráfico 1. Satisfação dos participantes acerca dos SRT. Botucatu (SP). Brasil, 2019

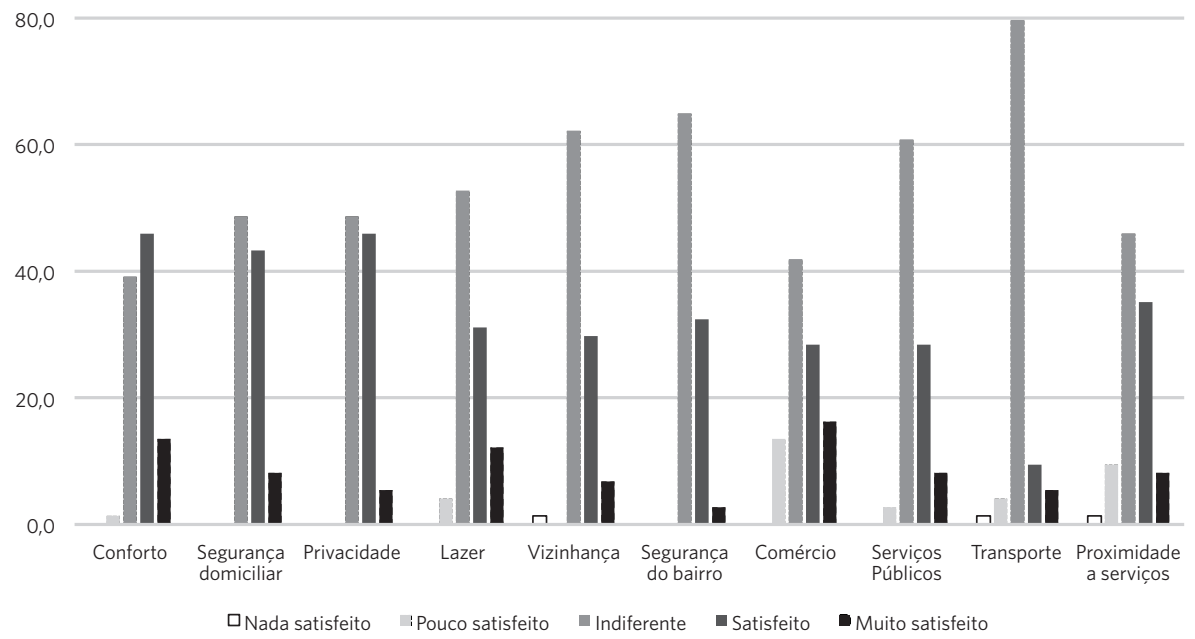

Fonte: Elaboração própria. 


\section{Discussão}

O perfil dos moradores dos SRT pesquisados aponta para faixa etária que caracteriza uma população idosa, com discreta predominância para o sexo feminino, sem parceiros e sem alfabetização. Em relação ao sexo, em uma população similar em um estudo do Brasil, encontrou-se predominância do masculino, diferindo dos achados desta pesquisa ${ }^{12-15}$. A faixa etária da população investigada neste estudo foi maior que a encontrada no perfil de moradores de SRT em outras regiões do Brasil12-15, em geral, com diferença de dez anos. São similares: o estado civil e a ausência de alfabetização ${ }^{12-14}$.

Observou-se que praticamente metade da amostra correspondeu a pessoas que receberam o diagnóstico psiquiátrico de esquizofrenia, seguido de retardo mental. Essa característica pode ser verificada também em outros estudos em que transtorno psicótico primário foi prevalente $(76,8 \%)^{13,16,17}$ e corresponde a uma herança do modelo asilar. Em uma população similar, a ocorrência de diagnósticos psiquiátricos também se centrou na esquizofrenia e no retardo mental ${ }^{\mathbf{1 4}}$, contudo, este último em menor proporção que o encontrado nesta pesquisa.

Ainda em relação ao psicodiagnóstico, observou-se uma quantidade de usuários institucionalizados em hospitais psiquiátricos que necessita ter melhor elucidação (35,8\%); essa população tem sido descrita com psicoses orgânicas não especificadas ou esquizofrenias outras ou não especificadas ${ }^{16}$. Diferentemente, nos resultados desta pesquisa, obteve-se a ocorrência de $12,16 \%$ (n=9) de psicodiagnósticos não especificados, contudo, essa porcentagem foi maior do que a encontrada em uma investigação com residentes de SRT do nordeste do Brasil ${ }^{14}$.

Importa ressaltar que 40,54\% da população $(n=30)$ apresentou apenas uma internação em hospital psiquiátrico, mantendo-se institucionalizada a partir dessa situação, sob tratamento medicamentoso e prática asilar.
Essa situação corrobora resultados de outros estudos ${ }^{15-17}$ e remete a discussão à centralidade do modelo manicomial no cuidado ao usuário com transtorno mental grave, associa-se à fragilidade de operacionalizar o cuidado de base comunitária e territorial, dispondo de serviços substitutivos suficientes na Raps; características próprias do modelo manicomial brasileiro que tem sido gradativamente desconstruído com a Reforma Psiquiátrica ${ }^{\mathbf{1 8}}$.

Outra faceta do modelo manicomial observada nessa população foi o impacto sobre as atividades do cotidiano, principalmente, as relações familiares. Isso se inicia ainda na internação psiquiátrica de pessoas com transtornos mentais graves, uma vez que uma parcela significativa $(74,4 \%, n=55)$ apontou não receber visitas ${ }^{16}$ - e perdura por toda sua permanência no aparato manicomial conforme reflexão de moradores de um SRT da região metropolitana de São Paulo ${ }^{19}$. Atenta-se para a pequena expressão de participantes $(12,16 \%, \mathrm{n}=9)$ que referiram receber visita de familiares no SRT, o que difere de outra pesquisa que registrou pouco mais da metade da amostra investigada ${ }^{\mathbf{1 4}}$.

O modelo manicomial produziu a exclusão da loucura da vida social, causando o distanciamento da cidade, da família, do lazer, do trabalho, da cultura e do direito à participação social, gerando a perda da condição de cidadania $^{20}$. Essa problemática prejudicou e prejudica as relações afetivas e trocas sociais, que são de extrema importância para a reabilitação psicossocial.

Os resultados desta pesquisa permitiram observar que os moradores do SRT2 têm maior percepção dos impactos atribuídos pelo transtorno mental grave em suas atividades cotidianas. Sob essa perspectiva, pode-se inferir que a saída do aparato manicomial com consequente restabelecimento no território, por meio da SRT, possibilita à pessoa com transtorno mental grave desenvolver a percepção das perdas psicossociais decorrentes do modelo manicomial.

Essa característica subsidia uma defesa intransigente acerca da inserção dos SRT nos espaços centrais da cidade. Em relação 
a esse posicionamento, os resultados encontrados entre os moradores do SRT1 e SRT2 apontaram, curiosamente, para características distintas: embora a localização geográfica do SRT2 seja favorável para inserção e autonomia por estar próxima de diversos serviços comunitários, no SRT1, estão alocados os moradores que mais recebem visita. Importa ressaltar que ambas as diferenças foram sustentadas por significância estatística.

A reinserção social deve considerar uma reconstrução possível, dentro do limite de cada sujeito. A conquista dos espaços do território é fundamental na construção do cenário de reinserção, podendo ser um espaço para experimentações que o encontro entre a loucura e a cidade pode oferecer ${ }^{21}$, avançando na dimensão sociocultural da Reforma Psiquiátrica, na medida em que mobiliza o imaginário social a fim de dirimir o estigma e preconceito em torno da loucura 1 .

Acerca da inserção social dos moradores dos SRT deste estudo, observou-se a centralidade desse dispositivo nos serviços de saúde. Concretamente, a exclusiva relação SRT e rede de atenção à saúde tornou-se evidente quando a totalidade referiu frequentar serviços de saúde e, concomitantemente, não frequentar serviços da educação, de segurança ou equipamentos religiosos. Conforma-se, com isso, uma ocupação social extremamente restrita e centralizada em serviços de saúde. Ao observar o perfil dos participantes, aponta-se para a necessidade de constituir o trabalho no SRT com base nos equipamentos intersetoriais.

A saída da instituição psiquiátrica e a chegada ao SRT, por si só, não garantem à pessoa com transtorno mental grave uma mudança real do modo de vida asilar, podendo reproduzir o cotidiano institucionalizante na casa, trancando-se em seus quartos, negando-se a participar das tarefas domésticas e permanecendo em uma situação de profunda passividade à espera de cuidados e tutela a que foram outrora submetidos ${ }^{8}$.

Com isso, constata-se a perpetuação e a renovação do modo manicomial nas práticas de cuidado comunitário produzindo uma grande dificuldade em tornar os SRT em moradias, uma vez que esses moradores são submetidos a regras múltiplas e dificilmente contam com espaços de privacidade ou distanciamento do olhar e da escuta alheios, sendo constantes a presença e o controle dos trabalhadores e dos colegas de moradia ${ }^{6}$.

Os SRT têm a característica de padronizar a disposição de móveis e objetos, principalmente pela preocupação dos cuidadores em manter a ordem no local, dificultando a escuta e o acolhimento de demandas que fujam à rotina. É evidente a existência de regras e valores morais que orientam moradores no modo como agem ou se comportam uns com os outros. O espaço de habitação requer plasticidade aos intentos de seus moradores e flexibilidade no suporte. Trata-se de uma diversificação não restrita à simples ampliação dos tipos predefinidos que irão impor ao sujeito e às suas necessidades específicas a construção de ideais externas ${ }^{6}$.

Observou-se que $81,1 \%(n=60)$ recebiam algum tipo de benefício do governo, contexto que indica a importância do $\mathrm{PVC}^{4}$ para os usuários do SRT, uma vez que todos os participantes da pesquisa utilizam todo o valor recebido para manter a moradia, promovendo condições de sobrevivência e maior possibilidade de inserção social. Por esse motivo a defesa à manutenção da garantia de direitos constituídos para as pessoas com transtornos mentais graves, representada pela dimensão político-jurídica da Reforma Psiquiátrica' ${ }^{1}$ que tem sido intensamente discutida haja vista as ameaças e os retrocessos sofridos desde o final do ano de $2015^{22}$.

Além da garantia institucional de obtenção do benefício financeiro, está a autonomia da pessoa com sofrimento psíquico grave em gerir seu próprio recurso ou uma parte dele. Esse direito representou um elemento importante para a sociabilidade dos moradores de SRT em pesquisa que observou que os pontos comerciais estavam entre os mais frequentados pela população estudada ${ }^{19}$. Situação inversa foi observada nesta investigação, em que se 
identificou controle excessivo sobre o acesso ao valor do benefício e um questionamento importante em relação ao destino dado ao auxílio recebido pelos moradores. Associado a essa situação, o distanciamento geográfico do centro comercial observado no grupo de moradores do SRT1 representa mais um elemento que fragiliza a produção de autonomia dos participantes deste estudo.

Considera-se que o passo inicial na recuperação de pessoas com transtorno mental grave, por meio da habitação, deve permear o trabalho e a educação, sem uma proteção excessiva dos cuidadores em todos os domínios da vida. Uma grande dificuldade é a impossibilidade de exercer um trabalho formal, pelo fato de receber o benefício, questão essa que não tira os sonhos e as expectativas de uma profissão, e impacta diretamente em um dos pilares da reabilitação psicossocial que corresponde ao trabalho com valor social ${ }^{8}$.

As estratégias de economia e cooperativas solidárias, equipamentos da reabilitação psicossocial da Raps ${ }^{2}$, são uma possibilidade para a questão do trabalho. O município desta pesquisa conta com uma cooperativa e uma oficina terapêutica voltadas para pessoas com transtorno mental grave, mas com mínima articulação com os moradores do SRT1. Nesses espaços, diversas oficinas voltadas para o trabalho e para a terapêutica são ofertadas; desse modo, além de permitir o trabalho com função social, a participação nesses serviços traz a potencialidade das trocas sociais e afetivas, desencadeando processos de subjetivação ${ }^{7}$.

A fragilidade na articulação pode ser apreendida em diversas situações: nos serviços da Raps entre si, nos SRT, e a intersetorialidade; bem como dos SRT com os diversos serviços de comércio, lazer e cultura. $\mathrm{O}$ isolamento dos SRT e sua desarticulação encaminham à reflexão sobre outra maneira de perpetuar a institucionalização da vida dos moradores, a qual, concomitantemente, atrofia a produção de subjetividades e de projetos de vida que se constroem à medida que se ocupa o espaço urbano ${ }^{19}$.
Como avaliação dos SRT deste estudo, está a satisfação dos usuários em relação a características como: conforto, segurança, privacidade, lazer, vizinhança, segurança comércio, serviços públicos, transportes e proximidade. Observou-se predominância para indiferença e satisfação, resultado que difere de outros estudos que apontaram elevada satisfação dos moradores acerca de algumas dimensões dos SRT, entre elas, as condições físicas e o conforto ${ }^{17,23}$. Identificou-se ainda que a satisfação dos participantes do SRT2 é maior quando comparada ao SRT1, diferença esta com significância estatística. Esses achados permitem argumentar sobre a importância de estar inserido e convivendo no território como um aspecto fundamental para a produção de implicação da pessoa com transtorno mental grave acerca do habitar 8

'Estar em casa' representa o direito de ir e vir, e um espaço de expressão de subjetividades, socialização, permitindo a potencialização da vida e da liberdade ${ }^{24}$. Passear quando desejar ou se quiser limpar um jardim, comprar mercadorias, descobrir ou redescobrir formas de se relacionar consigo e com outras pessoas, desenvolver um trabalho, seja ele remunerado ou não, fazer amizades, descobrir os direitos, e encontrar um namoro, ter a possibilidade de expressar a sua fé, que auxiliará a pessoa em sofrimento psíquico a resgatar a sua cidadania. O cuidado nos SRT caracteriza-se no trânsito do interno em direção ao externo: à rua, à vida ${ }^{8,25}$.

A realização deste estudo contribui para a discussão sobre a importância de os SRT operarem necessariamente na lógica intersetorial, como exercício para o desenvolvimento dos três pilares da reabilitação psicossocial, provocando a descaracterização desses serviços como pontos de atenção à saúde a fim de objetivar a desconstrução de práticas que perpetuem o modelo manicomial. Nesse rumo, apontam-se possibilidades de ação para os trabalhadores que atuam nesses serviços, considerando-os como objetos que estão alinhados aos objetivos da reabilitação psicossocial, o que 
implica, necessariamente, atos que extrapolem fazeres técnicos e especializados concentrados nos serviços de saúde.

O tamanho da amostra e o cenário de estudo representam os limites desta pesquisa e podem fragilizar possíveis generalizações dos resultados encontrados.

\section{Considerações finais}

Os resultados desta investigação permitem observar que os SRT do município estudado estão centralizados e se caracterizam exclusivamente como equipamentos de saúde. Tal característica dificulta a operacionalização do objetivo da residência terapêutica enquanto um meio para resgatar a dimensão do habitar própria da reabilitação psicossocial. Por consequência, a avaliação que as pessoas com transtornos mentais graves tecem acerca das necessidades e condições de moradia evidencia fragilizada implicação, mostrando-se indiferentes aos aspectos da inserção social por meio do habitar.

\section{Colaboradores}

Acebal JS (0000-0001-5438-6605)* contribuiu para a concepção e o planejamento, para a elaboração do rascunho e para a aprovação da versão final do manuscrito. Barbosa GC (0000-0002-7433-8237)* contribuiu para a revisão crítica do conteúdo e para a aprovação da versão final do manuscrito. Domingos TS (0000-0002-1421-7468)* contribuiu para a análise e interpretação dos dados, para a elaboração do rascunho e para a aprovação da versão final do manuscrito. Bocchi SCM (0000-0002-2188-009X)* contribuiu para a revisão crítica do conteúdo e para a aprovação da versão final do manuscrito. Paiva ATU (0000-0002-8457-628X)* contribuiu para a concepção e o planejamento e para a aprovação da versão final do manuscrito. 


\section{Referências}

1. Amarante P. Saúde Mental e Atenção Psicossocial. 3. ed. Rio de Janeiro: Fiocruz; 2007.

2. Brasil. Ministério da Saúde. Portaria $n^{\circ} 3.588$, de 21 de dezembro de 2017. Institui sobre a Rede de Atenção Psicossocial, e dá outras providências no âmbito do Sistema Único de Saúde (SUS). Diário Oficial da União [internet]. 21 Dez 2017. [acesso em 2021 jan 25]. https://bvsms.saude.gov.br/bvs/saudelegis/ gm/2017/prt3588_22_12_2017.html.

3. Brasil. Ministério da Saúde, Secretaria de Atenção à Saúde, Departamento de Ações Programáticas Estratégicas. Residências terapêuticas: o que são, para que servem. Brasília, DF: Ministério da Saúde; 2004.

4. Brasil. Ministério da Saúde, Secretaria de Atenção à Saúde, Departamento de Ações Programáticas Estratégicas. Programa de Volta pra Casa: liberdade e cidadania para quem precisa de cuidados em saúde mental. Brasília, DF: Ministério da Saúde; 2003.

5. Brasil. Ministério da Saúde. Portaria $n^{0} 3.090$, de 23 de dezembro de 2011. Dispõe, no âmbito da Rede de Atenção Psicossocial, sobre o repasse de recursos de incentivo de custeio e custeio mensal para implantação e/ou implementação e funcionamento dos Serviços Residenciais Terapêuticos (SRT). Diário Oficial da União [internet]. 23 Dez 2011. [acesso em 2021 jan 25]. Disponível em: http://bvsms.saude.gov.br/bvs/ saudelegis/gm/2011/prt3090_23_12_2011_rep.html.

6. Furtado JP, Tugny A, Baltazar AP, et al. Modos de morar de pessoas com transtorno mental grave no Brasil: Uma avaliação interdisciplinar. Ciênc. Saúde Colet. [internet]. 2013 [acesso em 2019 ago 15]; 18(12):3683-93. Disponível em: http:// www.scielo.br/scielo.php?script=sci_arttext\&pid $=$ S1413-81232013001200024.

7. Saraceno B. Libertando identidades. Da reabilitação psicossocial à cidadania possível. 2. ed. Rio de Janeiro: TeCorá; 2001.
8. Kantorski LP, Cortes JM, Guedes AC, et al. O cotidiano e o viver no Serviço Residencial Terapêutico. Rev Eletr Enf [internet]. 2014 [acesso em 2018 out 10]; 16(4):759-68. Disponível em: https://www.fen. ufg.br/revista/v16/n4/pdf/v16n4a08.pdf.

9. Furtado JP, Nakamura E. Inserção social e habitação de pessoas com sofrimento mental grave: um estudo avaliativo. São Paulo: Unifesp; 2014.

10. Sampieri RH, Collado CF, Lucio MPB. Metodologia de pesquisa. 5. ed. Porto Alegre: Penso; 2013.

11. Furtado JP, Moreira MIB, Nozabielle S, et al. Desenvolvimento de questionário dirigido às condições de moradia de usuários de CAPS. Saúde debate [internet]. 2013 [acesso em 2018 abr 5]; 37(96):62-75. Disponível em: http://www.scielo.br/pdf/sdeb/v37n96/08.pdf.

12. Alves C, Coutinho I, Reis T, et al. Perfil dos pacientes de três residências terapêuticas de um hospital psiquiátrico municipalizado do estado do Rio de Janeiro. Rev Pesq. [internet]. 2010 [acesso em 2019 out 5]; 2(supl):149-152. Disponível em: http://www.seer. unirio.br/index.php/cuidadofundamental/article/ view/848/pdf_105.

13. Lago EL, Machado RS, Vieira TS, et al. Perfil de moradores de serviços residenciais terapêuticos. Rev Enferm UFPI [internet]. 2014 [acesso em 2019 ago 25]; 3(1):10-7. Disponível em: https://revistas.ufpi. br/index.php/reufpi/article/view/1113/pdf.

14. França VV, Alves MP, Silva ALMA, et al. Quem são os moradores de residências terapêuticas? Perfil de usuários portadores de transtornos mentais desinstitucionalizados. Saúde debate [internet]. 2017 [acesso em 2019 out 5]; 41(114):872-84. Disponível em: http://www.scielo.br/scielo.php?pid=S0103$-11042017000300872 \&$ script=sci_abstract\&tlng=pt.

15. Soares LML, Silva PRF. Serviços Residenciais Terapêuticos na cidade do Rio de Janeiro: uma análise da estrutura e do processo de cuidado. Saúde debate [in- 
ternet]. 2019 [acesso em 2020 ago 16]; 43(spe7):10213. Disponível em: https://www.scielosp.org/article/ sdeb/2019.v43nspe7/102-113/.

16. Melo MCA, Albuquerque SGC, Luz JHS, et al. Perfil clínico e psicossocial dos moradores em hospitais psiquiátricos no estado do Ceará, Brasil. Ciênc. Saúde Colet. [internet]. 2013 [acesso em 2019 set 21]; 20(2):343-52. Disponível em: https://www. scielo.br/scielo.php?script=sci_arttext\&pid=S1413$-81232015000200343 \& \operatorname{lng}=$ en\&nrm=iso\&tlng=pt.

17. Oliveira WG. Avaliação da satisfação dos moradores com os Serviços Residenciais Terapêuticos [dissertação] [internet]. São João del-Rei: Universidade Federal de São João del-Rei; 2018. 94 p. [acesso em 2020 ago 15]. Disponível em: https://www.ufsj.edu.br/portal2-repositorio/File/ppgpsi/DISSERTACAO\%20 FINAL\%20Welley\%20Gouvea\%20oliveira.pdf.

18. Zanardo GLP, Moro LS, Ferreira GS, et al. Factors associated with psychiatric readmissions: a systematic review. Paidéia (Ribeirão Preto) [internet]. 2018 [acesso em 2019 out 19]; 28(21):e2814. Disponível em: https://www.scielo.br/scielo.php?script=sci_ arttext\&pid=S0103-863X2018000100202\&lng=en\&n $\mathrm{rm}=\mathrm{iso} \& \operatorname{tlng}=\mathrm{en}$.

19. Massa PA, Moreira MIB. Vivências de cuidado em saúde de moradores de Serviços Residenciais Terapêuticos. Interface Comun Saúde Educ [internet]. 2019 [acesso em 2020 ago 15]; 23:e170950. Disponível em: http://www.scielosp.org/article/icse/2019. v23/e170950/.

20. Amarante $\mathrm{P}$, Torre EHG. "De volta à cidade, sr. cidadão!” - reforma psiquiátrica e participação social: do isolamento institucional ao movimento antimanicomial. Rev Adm Pública [internet]. 2018 [acesso em 2019 set 27]; 52(6):1090-107. Disponível em: http:// www.scielo.br/scielo.php?script=sci_arttext\&pid=S 003476122018000601090\&lng=pt\&nrm=io.
21. Antonacci MH, Kantorski LP, Guedes AC, et al. O trabalho no serviço residencial terapêutico: possibilidades na reconstrução de vidas fora dos manicômios. Rev Pesq [internet]. 2017 [acesso em 2019 set 27]; 9(3):831-9. Disponível em: http://www.seer. unirio.br/index.php/cuidadofundamental/article/ view/5582.

22. Rossano CL. O avanço da Contrarreforma Psiquiátrica no Brasil. Physis [internet]. 2019 [acesso em 2019 set 27]; 29(1):e290101. Disponível em: https:// www.e-publicacoes.uerj.br/index.php/physis/article/view/43077.

23. Maluf R, Bandeira M, Oliveira D. Psychiatric patients' satisfaction in the therapeutic residence services: A positive experience of psychiatric deinstitutionalization. Estud Psicol [internet]. 2017 [acesso em 2020 ago 15]; 22(3):274-84. Disponível em: http://pepsic. bvsalud.org/scielo.php?script=sci_arttext\&pid=S1413$-294 X 2017000300004$.

24. Amorim AKMA, Dimenstein M. Desinstitucionalização em saúde mental e práticas de cuidado no contexto do serviço residencial terapêutico. Ciênc. Saúde Colet. [internet] 2009 [acesso em 2019 set 28]; 14(1):195-204. Disponível em: http:// www.scielo.br/scielo.php?script=sci_arttext\&pid $=$ S1413-81232009000100025.

25. Bessoni E, Capistrano A, Silva G, et al. Narrativas e sentidos do Programa de Volta para Casa: voltamos, e daí? Saúde Soc [internet]. 2019 [acesso em 2020 ago 15]; 28(3):41-53. Disponível em: https://www.scielo.br/scielo.php?pid=S0104$-12902019000300040 \&$ script=sci_arttext.

Recebido em 26/11/2019

Aprovado em 25/08/2020

Conflito de interesses: inexistente

Suporte financeiro: não houve 